\title{
ROUMANITÉ ET MÉDIATION CULTURELLE SOUS L'ANGLE DES MYTHES, DES LÉGENDES ET DES CONTES
}

\author{
Ciolăneanu, R. S.; Nanu, P. (ed.) (2020), Translation and Cultural Mediation \\ 2. Myths. Legends and Fairytales in Romania and across the World \\ Editions de l'Université de Turku, 328 p.
}

Roxana-Magdalena BÂRLEA ${ }^{1}$

\section{Introduction}

Les études roumaines s'enrichissent de nouvelles recherches, réunies dans un ouvrage publié chez Les Editions de l'Université de Turku : Ciolăneanu, R. S. ; Nanu, P. (ed.) (2020), Translation and Cultural Mediation 2. Myths. Legends and Fairytales in Romania and across the World, 328 p. ISBN ISBN 978-951-29-78854 (Print). ISBN 978-951-29-7886-1 (PDF) ${ }^{2}$.

Il s'agit du deuxième volume collectif d'une série prometteuse dont le " fil rouge » est le concept de médiation culturelle. Il vient donc dans la continuité de Translation and Cultural Mediation 1. Inside and Outside Perspectives on Romanian Language, Culture and Literature, paru en 2018 (mêmes coordonnateurs, même maison d'édition). La série met au centre l'enrichissement des connaissances et la consolidation des compétences interculturelles en passant par, notamment, la traduction ( We hold translation as one of the essential instruments that facilitate the contact between cultures and languages and, thus, mediates the intercultural communication and knowledge. », p. 9).

Structuré en 4 sections, l'ouvrage se présente sous la forme d'une collection de 17 articles, rédigés en anglais ou en roumain, autant de projections sur la problématique centrale de l'ouvrage. Certains d'entre eux sont des communications présentées au Colloque International RostUL8, organisé par La Faculté des Lettres de L'Université de Lisbonne en mai 2019 et présidé par Roxana Ciolăneanu, également coéditrice du volume. D'un côté, il comporte des recherches théoriques portant sur l'importance de se pencher sur la littérature d'enfance et de jeunesse ou la réception des textes appartenant à cette catégorie. D'un autre côté, il contient des

\footnotetext{
${ }^{1}$ Roxana-Magdalena Bârlea, Académie d'Etudes Economiques de Bucarest / Aix-Marseille Université / Institutul Limbii Române, roxana-magdalena.barlea@univ-amu.fr

${ }^{2}$ http://ficros.eu/wp-content/uploads/2020/04/Translation_and_Cultural_Mediation_ 2_2020.pdf
} 
études de cas qui mettent en rapport certains phénomènes de la culture roumaine avec des phénomènes similaires dans d'ailleurs, via des motifs, des symboles, des personnages emblématiques etc. Les auteurs abordent des thèmes tels que la mort, la figure du diable, les différents rituels et traditions populaires ou religieux, etc. Les concepts-clé sont le culturème, genius loci et la "couleur locale», car le volume se propose de mettre en évidence le dialogue des spécificités culturelles. Les corpus de ces recherches sont divers: des contes (populaires ou cultes, contemporains ou anciens), des fables, des légendes étiologiques, un livre d'Histoire pour le grand public et différents types de romans. Ceux-ci sont analysés dans la plupart des cas soit par le prisme des traductions du roumain vers une autre langue ou vice-versa, soit en les mettant en miroir avec des phénomènes correspondants dans d'autres contextes.

Les auteurs sont des enseignants-chercheurs provenant de différents établissements d'Allemagne, de Belgique, d'Espagne, de Finlande, d'Italie, de Portugal et de Roumanie. Un premier point fort de la publication est que les éditeurs ont lancé un appel à collaboration non seulement à des spécialistes confirmés, mais aussi à des (post)doctorants et à des chercheurs en début de carrière, dont les contributions sont à la hauteur de la tâche. C'est un choix éditorial méritoire, bénéfique pour la richesse du volume et porteur pour sa démarche pédagogique.

Le volume s'adresse aux «specialists in translation, cultural studies and intercultural communication, but also to linguists, language teachers, and everyone interested in informed and effective communication within and across cultures ", comme le déclarent les éditeurs dans l'avant-propos. Son architecture soutient cette pluralité de perspectives, car il est structuré de manière à faciliter différentes approches du texte dans sa globalité.

Dans le présent compte-rendu de lecture, parmi toutes ces catégories de récepteurs possibles, nous en privilégions deux, ie. les enseignants de culture roumaine et de roumain langue seconde (RLS), tout comme leurs étudiants. Ainsi, nous nous proposons de souligner ici la pertinence du volume en tant qu'outil de travail dans l'enseignement-apprentissage des langues-cultures, en l'occurrence du roumain en contexte européen. Notre objectif est donc de d'identifier et de décrire brièvement une sélection de thèmes et d'idées-clé qui peuvent être intégrés dans un cursus de RLS, afin d'indiquer des "entrées" possibles dans le volume, destinées à ce public-cible.

Si nous devions justifier notre choix, nous dirions seulement qu'actuellement, dans le domaine des études roumaines, la didactique n'est pas encore assez présente, par rapport aux recherches menées en littérature ou en linguistique. Pour ne donner qu'un seul exemple, les thèses d'habilitations qui portent sur la didactique en Roumanie ont du mal à se faire accepter et font des allers-retours entre la commission de philologie et celle de pédagogie - sciences de l'éducation. Néanmoins, la bonne nouvelle est que depuis quelques années, les colloques et les 
volumes collectifs de roumanistique comptent presque sans exception une section de didactique, qui est de plus en plus représentée en tant que nombre de communications et d'articles, ce qui ne peut qu'encourager les chercheurs à s'y pencher davantage.

En partant de la problématique générale, des idées principales de chaque auteur, des arguments qu'ils apportent, de leurs réflexions essentielles et de leurs conclusions, nous tentons de répondre à la question suivante : Comment faciliter l'accès des étudiants aux particularités de l'espace roumain en contexte européen dans un dialogue culturel ? Quel est le potentiel des traductions / adaptations et des comparaisons de différents types de textes lorsqu'on forme des médiateurs interculturels ? Ainsi, en partant des recherches théoriques, des études de cas et des récits d'expérience en classe de RLS, nous cherchons à distinguer les exemples (sources de connaissances ou modèles de pratiques de classe) qui peuvent être transposés dans d('autr)es contextes d'enseignement - apprentissage. Comme le sous-titre du volume l'indique, les articles portent sur « les mythes, les légendes et les contes, de Roumanie et d'ailleurs ». Dans les programmes de RLS, la place des mythes et des contes est indéniable, car ils font partie des «fondements » qui aident les apprenants à mieux comprendre un peuple. De plus, ils ont un excellent rôle formateur, comme le précise Ciolaneanu (p. 265) : «Stories (...) function as fundamental containers of ideas, beliefs and experiences used to build up solid identities and well-equipped individuals, fully able to effectively live and function in today's globalised and globalising society $»$. Dans ce qui suit, nous recensons les idées principales qui peuvent être intégrées dans un cours de RLS, telles qu'elles ressortent de ces articles.

\section{Traduction et médiation culturelle}

Neșu explicite les rapports entre les deux notions-clés - traduction et médiation culturelle - partagées par tous les auteurs :

(...) traducerea nu mai poate fi văzută și considerată ca un simplu fenomen lingvistic, ci devine, în mod obligatoriu, un amplu proces de mediere între două sau mai multe realități culturale - dezideratul final al oricărui parcurs didactic de predare/învățare a unei limbă străine. Ea ajută în mod activ la reconstituirea contextului cultural al unei limbi, permițând trimiteri, in funcție de tipologia textelor alese, la constituirea unui (sub)context cultural, istoric, literar etc., la activarea, umplerea și soluționarea gap-urilor culturale, a golurilor, sau a decalajelor dintre culturi. » (...) [Este] o activitate care presupune familiarizarea cu aspectele extralingvistice [ale unei limbi] și oferă avantajul contactului cu texte autentice (Neșu, p. 273).

Dans ce qui suit, nous allons jeter un bref regard sur ces deux concepts dans chacun des articles, que, pour notre compte-rendu, nous avons regroupés d'une manière différente de la structure du livre, en trois catégories thématiques : motifs 
universels dans la littérature roumaine ; traductions, adaptations, comparaisons de textes ; réflexions théoriques.

\subsection{Quelques motifs universels dans la littérature roumaine}

Quatre articles s'arrêtent sur des motifs et des thèmes universels et leurs concrétisations dans la littérature ou dans le folklore roumain.

Alice Toma aborde la circulation des motifs du mythe vers le conte populaire et vers la littérature culte et le thème de l'attitude devant la mort dans la culture roumaine / universelle. Elle part d'un chef d'œuvre de littérature culte, issu du folklore populaire, dont aucun cursus de RLS, quels que soient ses objectifs spécifiques, ne peut se passer; c'est peut-être LE conte roumain par excellence : Tinerețe fără bătrânețe și viață fără de moarte / Jeunesse sans vieillesse et vie sans mort (auquel fait référence également Nicolae - v. plus bas). Toma compare ce conte avec le roman fantastique de Mircea Eliade, Tinerețe fără tinerețe / Jeunesse sans jeunesse, qui y puise ses sources. Elle fait appel au concept de « christianisme cosmique » dans lequel Eliade trouve une spécificité du sud-est européen, afin de montrer comment se concrétisent dans la culture roumaine certains aspects liés à l'espace ou aux différents types de temps (physique, physiologique, affectif).

Un symbole universel riche en significations et particularisé dans les folklores locaux, le serpent, est approfondi par Ioana-Ruxandra Fruntelată, qui adopte les principes théoriques de l'ethnologie pour comparer des légendes étiologiques portugaises et roumaines. Elle s'appuie sur le concept de genius loci pour mettre en parallèle les Mouras encantadas, associées à des mégalithes portugais et les légendaires fées qui veilleraient sur les maisons roumaines ou sur les travailleurs dans les mines de Roșia Montana, en Transylvanie. Les deux types de personnages surnaturels se montrent sous la forme de serpents pouvant se métamorphoser en de belles femmes ; ils ont aussi deux côtés antinomiques (protecteurs / dangereux). Au delà de ces points communs, l'auteur discute les différences culturelles, identifiées dans les pratiques populaires autour de ces esprits et les fonctions qui leurs sont attribuées dans chacun des deux espaces. C'est une interprétation comparative ethnologique qui encourage les étudiants à mieux connaitre non seulement la culture-cible, mais aussi leur propre patrimoine folklorique.

Mircea Păduraru présente une contribution portant sur l'image du Diable dans l'imaginaire collectif contemporain des Roumains, tel qu'il apparait dans la «mythologie diffuse » (dans le sens de Hedesan 2000), en tant qu'héritage des croyances religieuses (à propos d'apparitions et de rituels de purifications), dans les expressions figées du langage courant et dans la publicité. L'auteur conclut: « the Romanian cultural model appears to be heavily dependent on the Devil's structuring powers and on the distinctions and nuances that it brings about. A deliver from the evil one is anywhere than near or imminent. »(p. 206). Ses exemples méritent d'être exploités dans une perspective dialogique interculturelle.

SYNERGY volume 17, no. $2 / 2021$ 
Le chapitre coécrit par Irina David et Anca-Teodora Șerban-Oprescu contribue à expliquer le rôle des fables dans la construction du mental collectif des Roumains. Il y a ici un double intérêt pour les apprenants de roumain langue étrangère. D'une part, ils ont accès à une synthèse de ce genre dans la littérature roumaine (apparition relativement tardive par rapport aux grands modèles européens, thématique tributaire à ceux-ci et principaux représentants). D'autre part, l'analyse des fables a un apport considérable au tableau de la société roumaine du XIX ${ }^{\text {ème }}$ siècle. Comme elles atteignent le maximum de prolifération en Roumanie à l'époque de la libération de l'influence Ottomane, les fables comptent parmi les «armes» des Roumains illuministes pour faire passer leurs idées révolutionnaires pendant cette période de modernisation. Dans ce contexte, l'universalité des thèmes traités par les fables nationales se voit, par exemple, dans le fait que le mécontentement des Roumains par rapport aux problèmes sociaux du moment s'encadre dans un mouvement européen similaire. L'un des sujets favorisés chez nous est la critique des inégalités sociales et du manque de scrupules observé chez certains individus lors de leur ascension sociale. David et ȘerbanOprescu mettent l'accent sur ce qui était acceptable et ce qui ne l'était pas dans la société à ce moment-là. Un bon exemple est un vers de fable devenu expression figée, encore utilisée de nos jours, que les auteurs citent : (Că) voi egalitate, dar $n u$ pentru căței. / Je veux l'égalité, mais pas pour les chiots (Le chien et le chiot, Gr. Alexandrescu, 1842). Cette expression, qui renvoie de manière ironique à ceux qui prêchent l'égalité, mais ne la pratiquent pas, se retrouve actuellement dans le discours quotidien ou dans les articles de presse (à propos des lois qui s'appliquent seulement aux citoyens ordinaires et non pas aux privilégiés). Ainsi, la lecture de ce chapitre aide les étudiants étrangers à comprendre également certains aspects relevant de l'intertextualité dans la culture roumaine.

\subsection{Traductions / adaptations et comparaisons de textes}

Les traductions / adaptations de textes, notamment littéraires, du roumain en italien, allemand, anglais, japonais, mais aussi de l'anglais ou du finnois vers le roumain, et les problèmes (techniques ou de réception) qu'elles posent font l'objet de 10 chapitres du livre.

D'un côté, les auteurs traitent les provocations linguistiques que les traducteurs rencontrent. Travaillées dans des ateliers spécialisés de traduction ou comme complément aux cours de RLS, ces difficultés sont un exercice approprié surtout pour les niveaux $\mathrm{C} 1$ et $\mathrm{C} 2$, dans le contexte actuel où l'on constate un retour de la traduction en tant que stratégie d'apprentissage en didactique des langues. D'un autre côté, sont mis en évidence les éléments de signification propres à une culture et la manière de les transposer dans un autre espace, dont les coordonnées géographiques, esthétiques, religieuses, mythologiques, pragma-linguistiques, comportementales etc. sont différentes. C'est une approche interculturelle qui devrait faire partie de tout programme de RLS. Les cadres théoriques passent par les principes de la linguistique intégrale, développés par Coșeriu entre 1973 et

SYNERGY volume 17, no. 2/2021 
1996 (Nicoleta Neșu), les culturèmes et leur classification par Molina Martinez, 2006 (Harieta Topoliceanu), le concept de «traduction dialogique» chez O’Sullivan, 2005 (Daniela Hăisan), l'esthétique de la présence de Nancy, 2004 (Valeriu Stancu), etc.

Sept articles ont comme point de départ des contes roumains, examinés sous l'angle de leurs particularités dans la littérature universelle, leur traduction en différentes langues ou encore leur comparaison avec des contes similaires circulant dans d'autres cultures.

Raluca Nicolae s'intéresse aux difficultés qu'entrainent la traduction en japonais du conte Tinerețe fără bătrânețe și viață fără de moarte / Jeunesse sans vieillesse et vie sans mort. Elle regarde de près le «voyage» de ce texte entre les deux espaces, en s'arrêtant sur la métaphore du titre - déjà un univers en soi -, les formules type du conte, certains personnages dont les caractéristiques sont culturellement marquées ou encore l'expression de la politesse dans les formules d'adresse. La médiation passe cette fois-ci par des concepts tels que l'immortalité ou les tabous, à explorer dans un discours où le traducteur joue le rôle de « négociateur des cultures » (Roth, 1998).

D'autres contes fondamentaux dans la construction de l'identité roumaine, à intégrer absolument dans un curriculum de RLS, sont analysés par Harieta Topoliceanu et Nicoleta Neșu, par le biais de leurs traductions en italien. Il s'agit de trois des contes cultes d'inspiration populaire les plus connus, écrits par deux des « piliers » de la culture roumaine, Ion Creangă et Mihai Eminescu.

Topoliceanu évoque la Roumanie rurale du XIX ${ }^{\text {ème }}$ siècle. Elle part du concept de « culturèmes » et de leurs catégorisations par Molina Martinez, 2006, afin de mettre en valeur les solutions identifiées par les traducteurs (adaptations, traductions mot-à-mot, expressions équivalentes, notes explicatives, etc.) pour faciliter l'accès du lecteur italien à certaines réalités roumaines de l'époque. C'est une très bonne méthode d'exposer les étudiants en RLS à des éléments du patrimoine immatériel tels que les rituels religieux et populaires liés au basilic ou encore les croyances autour du mariage des jeunes filles (cf. l'expression « piatră de moară în casă », en référence à la difficile et hautement importante tâche de marier ses jeunes filles). Travailler différentes catégories de culturèmes en classe, par l'intermédiaire de la lecture en parallèle des textes en original et de leurs traductions, notamment érudites, accompagnées de notes de bas de page et d'explications, est une excellente mise en pratique du dialogue des civilisations avec les étudiants. C'est une étape essentielle dans le processus d'appropriation de la culture-cible, mais aussi une activité de réorganisation concernant les représentations qu'ils ont de leur propre culture. De plus, l'article de Topoliceanu peut être proposé aux apprenants avancés comme exercice pour observer les différences de registre à un moment précis de l'évolution des deux langues mises en parallèle. 
Neșu s'occupe de la traduction dans le champ de recherche de la linguistique contrastive et traite un conte roumain, en comparant la variante originale avec l'une de ses traductions en italien qui est précédée d'une étude explicative. Elle discute le choix de la traductrice de garder en original les noms de personnages emblématiques (Făt-Frumos, Ileana Cosânzeana) ou des culturèmes tels que doine et hore. Par ailleurs, le mot doină (pluriel, doine) est même inscrit dans le patrimoine UNESCO, ensemble avec deux autre mots (dor et colind) faisant référence à des traditions roumaines ; il n'est donc pas étonnant que sa traduction est problématique. Sa contribution s'inscrit déjà dans une visée didactique, car elle scrute ces textes avec ses étudiants en RLS dans un laboratoire de traduction. Un choix qui invite l'enseignant de langue-culture roumaine à échafauder tout un scenario didactique de reconstitution des multiples significations de ces personnages et des fonctions qui leurs sont attribuées, en s'appuyant sur les connaissances des étudiants liées aux théories du conte et aux phénomènes similaires présents dans leur culture.

Alexandra Cherecheș va vers un «roman » autobiographique roumain fascinant, difficilement accessible pour un locuteur non-natif du fait de l'oralité de son style : Amintiri din copilărie / Souvenirs d'enfance, d'Ion Creangă - une vraie provocation en classe de RLS. Son chapitre met en parallèle certaines pratiques et croyances du monde rural du XIX ${ }^{\mathrm{e}}$ siècle avec des traditions et des superstitions correspondantes dans d'autres cultures. Un exemple est l'organisation de șezători, réunions de « socialisation» à l'ancienne, où les jeunes filles et les jeunes hommes passaient du temps ensemble, chantaient, racontaient des histoires, mais aussi flirtaient et trouvaient un partenaire de vie dans un contexte accepté par les normes sociales de l'époque et dont le cadre était très ritualisé. L'auteur en trouve des équivalents dans le folklore espagnol, mais ils existent aussi chez d'autres peuples. Les sujets abordés dans cet article méritent des développements plus amples, à explorer avec les étudiants, en perspective comparée.

Brândușa Grigoriu s'attache à identifier la "couleur locale » dans la version roumaine de l'histoire d'Alexandre le Grand, en mettant l'accent sur la forme que prend le mythe de la chute du Paradis dans Codex Neagoeanus (1620). Le chapitre est très utile pour ceux qui étudient le processus d'adaptation de ce texte dans les cultures des Balkans et dans l'espace roumain. L'œuvre est représentative pour la manière dont les idées et le style appartenant au fond local se greffent sur l'histoire. Elle est remarquable aussi pour la manière dont elle a influencé à son tour la littérature roumaine, tant dans ses concrétisations orales-populaires que dans la création culte, chez de nombreux écrivains.

Adina Vladu compare un conte contemporain (Ninigra și Aligru) avec sa variante en anglais (Tigrino and Tigrene), en auto-traduction / auto-adaptation. La clé de voûte est ici la manière dont Cassian réajuste une histoire de la Roumanie communiste pour le public américain. Vladu observe la manière dont Cassian réécrit sont texte, notamment à l'intention des adultes, en explicitant les allusions politiques, voilées dans la version roumaine pour qu'elles échappent à la censure, et en ajoutant des références culturelles, afin d'expliquer le contexte politique et les subtilités du double langage utilisé dans la première version. 
Nicoleta Neșu et Valeriu Stancu restent dans le domaine de la traduction, mais changent de type de texte. Leurs chapitres évoquent des pratiques liées à des ateliers de traduction, parfaitement transposables dans d'autres contextes d'enseignement/apprentissage.

Neșu part d'un livre d'Histoire pour le grand public (Cioroianu, 2013), doublé d'une émission de télévision (Ifrimache, Ioniță, 2013-2018, présentée par Cioroianu) que de nombreux enseignants de roumain langue étrangère utilisent avec leurs apprenants, notamment pour illustrer des contenus culturels. Elle utilise ce document authentique dans un exercice de traduction, reliant le texte écrit en roumain et sa version vidéo à la traduction en italien, réalisée par ses étudiants. Ainsi, à la perspective culturelle, utilisée d'habitude par les enseignants pour les « histoires » de Cioroianu, Neșu ajoute l'examen contrastif au niveau textueldiscursif et grammatical.

Stancu se consacre à un roman contemporain et au transfert du sens esthétique de la version originale en roumain à sa traduction en allemand. La technique qu'il utilise avec ses étudiants, tout comme la manière décrite pour mettre en pratique la théorie esthétique de la «présence», de travailler sur la reconstitution de « l'atmosphère », de la " charge culturelle» au niveau linguistique et contextuel mènent naturellement à sa conclusion, qui s'ouvre sur tout un programme de recherche en esthétique, basée sur la traduction littéraire.

Deux autres chapitres sont dédiés à des textes de littérature d'enfance anglaise et finnoise et leurs traductions en roumain. La réception des textes appartenant à d'autres littératures européennes en Roumanie peut également être un cheminement intéressant pour les étudiants qui veulent comprendre certaines « nuances » de l'identité roumaine.

Daniela Hăisan traite d'une série de contes de la littérature anglaise (Goth Girl, de Chris Riddell), traduits en roumain. Dans cet article nous retenons notamment l'explicitation des jeux de mots, de l'intertextualité, des mots " porte-manteaux », des proverbes, des citations, des allusions culturelles etc. qui font appel à des connaissances extralinguistiques pour créer des effets d'humour. Un matériel adéquat pour un cours de traduction pour les étudiants avancés.

Paul Nanu tente une explication pour l'échec en Roumanie d'un succès littéraire international. Il s'agit d'une série de contes finnois dont les personnages sont devenus une vraie «marque pays » et attirent des touristes étrangers en Finlande (Moomins, de T. Jansson). Les histoires des Moomins, best sellers à travers le monde, aident des millions d'enfants et d'adultes à découvrir la vie quotidienne dans « le pays des mille lacs", des normes et des coutumes locales, mais aussi à réfléchir à des problèmes sociaux. Pourtant, en Roumanie elles ne connaissent que quelques traductions, sans interventions explicatives, et restent presque inconnues du grand public et de la critique. La cause en est probablement le manque de proximité entre les deux cultures, ce qui montre bien que le silence a aussi sa signification dans dialogue culturel. 
Trois articles ne s'appuient pas sur des exemples particuliers, mais fixent un cadre plus général. Pourtant, puisque la plupart des contributions de ce volume (onze sur dix-sept) sont consacrées à la littérature d'enfance, ces trois pièces complètent le puzzle, en rappelant la nécessité de voir comment le concept de «médiation culturelle » se reflète dans ce «secteur» littéraire, qui soulève des débats (cf. Nières-Chevrel, 2017). Ces articles s'inscrivent donc dans une lignée de recherches très actuelles et offrent des suggestions de scénarios à utiliser par la suite dans des cours de culture roumaine.

Roxana Ciolăneanu souligne le rôle que joue ce type de littérature dans la consolidation des connaissances sur le monde, des valeurs et des comportements acceptés dans des espaces culturels spécifiques. Son article se focalise notamment sur l'éducation des enfants, mais les principes énoncés sont parfaitement valables également pour notre public-cible. Nous retenons de cet article au moins deux idées fondamentales à mettre en pratique avec eux. Tout d'abord, l'idée de mettre la compétence interculturelle, basée sur différents principes théoriques, parmi lesquels ceux de Byram, 2008, au centre de la démarche éducative. Et ensuite l'optique axée sur les concepts de story et de storytelling / narration, conte - pour développer chez l'apprenant, dès le plus jeune âge, la curiosité, l'esprit de découverte et la compréhension de points de vue multiples sur un même phénomène. Ainsi, Ciolăneanu propose une stratégie basée sur six questions clés qui a comme objectif d'amener l'apprenant à comprendre les «autres» et se comprendre soi-même, à mieux écouter et à communiquer de manière plus efficace, bref, à devenir un médiateur culturel actif.

Gabriel Nedelea parle de la «matrice romantique » du « lecteur idéal» dans la littérature de jeunesse et de la manière dont sont construites les narrations à propos de l'enfance, en passant par quelques-uns des œuvres phares de la littérature universelle. Un raisonnement à poursuivre également pour la littérature roumaine.

Sébastien Chonavey expose son propre ouvrage (Dis c'est quoi les théories du Complot?, 2019), conçu comme un livre de popularisation à l'intention des adolescents, écrit par un enseignant. Il met en relief les différentes catégories de difficultés que pose ce type d'écriture (nature du sujet, objectifs, notions utilisées, registre stylistique, etc.) et les solutions qu'il a identifiées, dont il explique le choix. C'est une analyse réflexive qui nourrit le débat autour de la littérature d'enfance.

\section{Conclusion}

Nous avons très brièvement présenté la manière dont le volume Ciolăneanu, R. S.; Nanu, P. (ed.) (2020), Translation and Cultural Mediation 2. Myths. Legends and Fairitales in Romania and accross the World, Les Editions de 
l'Université de Turku met en avant un dialogue entre la culture roumaine et des cultures spécifiques (allemande, anglaise, espagnole, italienne, finnoise, française, japonaise et portugaise), au travers du concept de médiation. Le bilan de l'ouvrage est sans nul doute positif. En empruntant des voies complémentaires, les auteurs répondent au problème posé en avant-propos. C'est une réflexion importante, qui contribue à mieux faire connaître la roumanité et à en préciser sa place dans la culture universelle.

Sont intérêt est certain pour plusieurs catégories de lecteurs, parmi lesquels nous avons mis l'accent sur les apprenants étrangers, dont l'objectif est de comprendre l'imaginaire collectif et l'identité des Roumains. Les différentes approches peuvent servir de modèles tant aux enseignants de RLS qu'à leurs étudiants, qui peuvent s'y référer afin de compléter leur documentation pour préparer différents sujets d'examen ou de dissertation.

Pour élargir le champ de ses débouchés, ce volume peut inspirer un cours à l'intention des étudiants étrangers intitulé «Culture roumaine - culture européenne. Une perspective comparée basée sur des mythes, des légendes et des contes ».

Nous espérons que la série continuera et qu'un troisième volet de cette série, pourquoi pas axé de manière explicite sur la didactique des langues-cultures, verra bientôt le jour.

\section{Bibliographie}

Byram, M. 2008. From Foreign Language Education to Education for Intercultural Citizenship. Essays and Reflections, Clevedon/Buffalo/ Toronto: Multilingual Matters.

Cioroianu, A. 2013. Cea mai frumoasă poveste. Câteva adevăruri simple despre istoria românilor, București: Curtea Veche.

Hedesan, O. 2000. Pentru o mitologie difuză, Timișoara: Marineasa.

Ifrimache, I., Ioniţă, D. (producteurs). 2013-2018. 100 de poveşti despre Marea Unire - 5 minute de istorie, émission TVRInternational présentée par A. Cioroianu.

Molina Martinez, L. 2006. El otoño del pingüino, Castellón: Universidad Jaime I.

Nancy, J.-L. 2004. "Das Bild: Mimesis \& Methexis", in Godzich, W.; Huber, J. (eds.), Ästhetik Erfahrung, Wien / Zürich: Springer / Voldemeer.

Nières-Chevrel, I. 2017. "Quand l'Université se saisit de la littérature d'enfance et de jeunesse", in Strence [En ligne], 12 / 2017. DOI: https://doi.org/ 10.4000/strenae.1716. Retrieved from http://journals.openedition.org/ strenae/1716. Accessed on 23 May 2021.

O'Sullivan, E. 2005. Comparative Children's Literature, London and New York: Routledge.

Roth, K. 1998. "Crossing Boundaries: The Translation and Cultural Adaptation of Folk Narratives", in Fabula, vol. 39 (3/4): 243-255. 